\title{
Oxygen in patients with fibrotic interstitial lung disease: an international Delphi survey
}

\author{
Rachel K. Lim ${ }^{1}$, Christopher Humphreys ${ }^{1}$, Julie Morisset ${ }^{2}$, Anne E. Holland $\mathbb{1}^{3,4}$, \\ Kerri A. Johannson $\circledast^{1,5}$ and the $\mathrm{O}_{2}$ Delphi Collaborators ${ }^{6}$ \\ Affiliations: ${ }^{1}$ Dept of Medicine, University of Calgary, Calgary, AB, Canada. ${ }^{2}$ Dept of Medicine, Centre \\ Hospitalier de l'Université de Montréal, Montréal, QC, Canada. ${ }^{3}$ Dept of Physiotherapy, La Trobe University, \\ Melbourne, Australia. ${ }^{4}$ Dept of Physiotherapy, Alfred Health, Melbourne, Australia. ${ }^{5}$ Dept of Community Health \\ Sciences, University of Calgary, Calgary, $\mathrm{AB}$, Canada. ${ }^{6} \mathrm{~A}$ list of the $\mathrm{O}_{2}$ Delphi Collaborators can be found in the \\ acknowledgements section.
}

Correspondence: Kerri A. Johannson, South Health Campus, 4448 Front Street S.E., Calgary, AB T3M 1M4, Canada. E-mail: kerri.johannsondahs.ca

@ERSpublications

Supplemental oxygen should be recommended for patients with fibrotic interstitial lung disease in cases of severe resting hypoxaemia, or exertional hypoxaemia particularly with attributable symptoms or exercise limitation http://bit.ly/2Hsq6sq

Cite this article as: Lim RK, Humphreys C, Morisset J, et al. Oxygen in patients with fibrotic interstitial lung disease: an international Delphi survey. Eur Respir J 2019; 54: 1900421 [https://doi.org/10.1183/ 13993003.00421-2019].

\section{ABSTRACT}

Rationale: Patients with fibrotic interstitial lung disease (ILD) frequently develop resting or exertional hypoxaemia. There is heterogeneity in clinical practice and a paucity of evidence guiding supplemental oxygen use in this patient population. The objectives of this study were to build international expert-based consensus on the indications and goals of supplemental oxygen from the perspective of healthcare providers, and identify potential barriers to its access.

Methods: Semistructured interviews and a comprehensive literature search informed items for the Delphi survey, with items not meeting consensus included in round 2. Round 3 contained survey questions regarding regional funding coverage for oxygen therapy. A priori definitions of consensus were median scores of 4 (agree) to 5 (strongly agree) for "agreement", 1 (strongly disagree) to 2 (disagree) for "disagreement" or 3 (unsure) with an interquartile range of $0-1$.

Results: 42 out of 45 (93\%) experts completed all three survey rounds, representing 17 countries. 20 out of 36 items met consensus for agreement or disagreement, 10 items met consensus for unsure and four items did not meet consensus. Experts agreed that oxygen should be recommended for patients with severe resting hypoxaemia and in cases of exertional desaturation to $<85-89 \%$, particularly with attributable symptoms or exercise limitation. There are regional differences in funding coverage for oxygen, based on desaturation thresholds, clinical symptoms and testing requirements.

Conclusions: Experts achieved consensus on 20 items guiding supplemental oxygen use in fibrotic ILD. These findings may inform research, clinical recommendations and funding policy.

Published online 8 Aug, 2019; republished 20 Jan, 2020 to acknowledge the collaborator group in the author line.

This article has supplementary material available from erj.ersjournals.com

Received: Feb 282019 | Accepted after revision: May 042019

Copyright OERS 2019 


\section{Introduction}

Interstitial lung disease (ILD) represents a large and heterogeneous group of disorders that can lead to progressive symptoms of dyspnoea, cough and exercise intolerance. Among those with advanced fibrotic ILD, hypoxaemia is common. Impaired gas exchange develops due to ventilation-perfusion mismatching, diffusion limitation and vascular abnormalities [1-3]. Exertional hypoxaemia, in the absence of resting hypoxaemia, is a common characteristic of fibrotic ILD that is often more severe than in other lung diseases such as chronic obstructive pulmonary disease (COPD) [4]. In addition, both exertional and resting hypoxaemia have been associated with increased mortality in this patient population $[5,6]$.

Important patient-centred priorities for individuals with fibrotic ILD include improving dyspnoea, exercise capacity and health-related quality of life [7]. Supplemental oxygen is commonly prescribed in routine clinical practice with these goals in mind; however, robust evidence to support its long-term use is lacking. Several small studies of short-term oxygen use in patients with ILD have shown that it can improve dyspnoea, endurance and walking distance [8-10]. However, other studies have failed to identify symptomatic or physiological benefits $[11,12]$. Data in patients with isolated exertional hypoxaemia are limited, but a recent randomised crossover trial demonstrates that short-term oxygen use is associated with improved health-related quality of life in patients with fibrotic ILD [13]. No studies have adequately evaluated the role of long-term oxygen on outcomes such as disease progression or mortality in ILD, in either resting or ambulatory hypoxaemia. Recommendations for long-term oxygen therapy for resting hypoxaemia in ILD are largely extrapolated from trials conducted in COPD, where a survival benefit is well established $[14,15]$. Clinical guidelines on the management of idiopathic pulmonary fibrosis (IPF) and other ILDs are also subject to limitations imposed by current gaps in knowledge. While most recommend oxygen therapy for patients with IPF and resting hypoxaemia [16-18], there are inconsistent recommendations for other common clinical scenarios such as isolated exertional hypoxaemia.

Given the paucity of data, it is unknown which fibrotic ILD patients are most likely to derive benefit from supplemental oxygen and in which clinical scenarios. This contributes to important variability in prescribing practices and funding coverage, including pre-testing algorithms that differ geographically due to regional infrastructure [19]. We conducted an international Delphi survey in an effort to develop a consistent best practice approach to supplemental oxygen use in patients with fibrotic ILD. The objectives of this Delphi study were to build expert-based consensus on the indications and goals of supplemental oxygen therapy, and to identify potential barriers to its prescription and funding coverage. This study specifically focused on these goals from a healthcare provider's perspective, and was not designed to address the perspectives and experiences of patients living with fibrotic ILD. Some of these results were presented in abstract form at the 2019 American Thoracic Society International Conference [20].

\section{Methods}

This study was approved by the Conjoint Health Research Ethics Board of the University of Calgary (Calgary, AB, Canada) (approval REB17-1669_REN1) and all participants provided informed consent.

\section{Identification of Delphi survey items}

One investigator (K.J.) conducted individual, semistructured telephone interviews with recognised ILD experts, selected based on research experience in ILD and supplemental oxygen, and representing diverse practice regions. Open-ended questions regarding the indications, goals and practical challenges of oxygen use in ILD patients were posed to all participants (see interview guide in the supplementary material). Interviews were transcribed and analysed by two investigators (R.L. and C.H.) using a content analysis approach [21, 22]. Results of the qualitative interviews and a comprehensive literature review informed survey items for the first Delphi round.

\section{Participant panel selection}

Panellists were identified through contacts from the professional networks of K.J., A.H. and J.M. or through publication and citation records. We aimed for an international group of ILD experts with a range of clinical and research experience, representing a diversity of geographical practice locations. An initial list of potential participants with their region and country of practice was created. Aiming to achieve representation in age, sex, healthcare provider profession and career stage, the initial list was culled. The final list had proportionally greater representation from larger geographical regions (e.g. the USA) or smaller regions with an established track record of publications on the specific study content (e.g. Australia and the UK). The identified experts, including those who participated in the qualitative interviews, were invited to participate in the first round of the Delphi survey.

\section{Delphi survey execution}

The Delphi study was conducted in accordance with the reporting standards developed in CREDES (Conducting and REporting of DElphi Studies) [23]. Surveys were disseminated and completed online 
using Qualtrics software (www.qualtrics.com). Surveys were administered $\sim 3$ weeks apart and participants were given 2 weeks to complete their responses. E-mail reminders were sent to maximise participation rates and participants not completing a round of the survey were not invited to subsequent rounds. Items in the first two rounds were rated using a 5-point Likert scale, where items were rated as 1 (strongly disagree), 2 (disagree), 3 (unsure), 4 (agree) and 5 (strongly agree). The a priori definitions of consensus were median scores of 4-5 for "agreement" and 1-2 for "disagreement" with an interquartile range (IQR) of $0-1$. A median score of 3 with an IQR of 0-1 was considered consensus for "unsure". These definitions were chosen to represent the spread of responses for each item [24]. Items not meeting consensus in round 1 were repeated in round 2, while items meeting consensus were removed from subsequent surveys.

In round 1, participants could provide feedback and suggest additional survey items for round 2, with responses reviewed to identify original items within the scope of the study for subsequent inclusion. If deemed appropriate, round 1 survey items were revised for clarity prior to dissemination in round 2 . During round 2, participants were given the distribution of group answers for survey items repeated from round 1 and provided new survey items. They also answered one multiple choice question about the peripheral oxygen saturation $\left(\mathrm{S}_{\mathrm{PO}_{2}}\right)$ threshold below which they recommend exertional supplemental oxygen, a suggestion from round 1. In round 3, participants were asked about regional funding policies for patients meeting the indications for supplemental oxygen use, as defined by consensus from prior rounds. Additional questions focused on the availability of clinical investigations agreed upon by earlier consensus. Answers were "yes" or "no", but participants could provide optional text.

\section{Statistical analysis}

Descriptive statistics were used to characterise the study participants. Quantitative group results for each item (median) and the level of dispersion (IQR) were calculated to identify items meeting the a priori definitions of consensus for agreement, disagreement or unsure after rounds 1 and 2. A sensitivity analysis was conducted using an alternative method of determining consensus, defined as $\geqslant 70 \%$ agreement for scores of either 1 or 2,4 or 5 , or 3 . Results from round 3 were summarised descriptively. Statistical analyses were performed using Stata version 15.1 (StataCorp, College Station, TX, USA).

\section{Results}

\section{Expert survey participants}

10 out of $10(100 \%)$ invited experts participated in the semistructured individual interviews (table 1). A total of 45 ILD experts were invited by e-mail to participate in the Delphi survey, including those completing the interviews, with 42 out of $45(93 \%)$ completing all three rounds. The survey participants had a mean \pm SD age of $50.5 \pm 8.5$ years, 19 (45\%) were female, 39 (93\%) were physicians, three (7\%) were nurses, with a mean \pm SD of $19.1 \pm 9.3$ years in independent clinical practice and $77.3 \pm 22.3 \%$ of clinical time dedicated to patients with ILD. Experts represented 17 countries and five continents.

\section{Survey items}

Based on the semistructured expert interviews and literature review, 32 items were included in the first round of the survey (figure 1). 23 items met consensus and were excluded from round 2. Nine items were repeated in round 2, with four new items. After round 2, eight items met consensus and four did not. The round 3 survey contained 16 questions regarding regional funding criteria and access to supplemental oxygen and physiological testing for patients with fibrotic ILD.

\section{Delphi survey results}

A total of 18 items met consensus for agreement and two met consensus for disagreement (table 2). All experts strongly agreed that oxygen should be recommended in cases of severe resting hypoxaemia (i.e. arterial oxygen tension $\left(\mathrm{PaO}_{2}\right)<55 \mathrm{mmHg} / \mathrm{SpO}_{2}<89 \%$, or $\mathrm{PaO}_{2}<60 \mathrm{mmHg}$ and cor pulmonale and/or polycythaemia). There was consensus that oxygen be recommended with the goals of addressing a broad range of symptoms, functional impairments and/or physiological derangements. There was consensus that resting hypoxaemia leads to pulmonary hypertension in patients with fibrotic ILD and that the development or worsening of exertional hypoxaemia provides evidence of clinical deterioration. There was consensus that patients with exertional hypoxaemia should not be advised to use oxygen during sleep without objective testing documenting nocturnal desaturation and that all patients should undergo such screening tests. All experts strongly agreed that newer portable oxygen delivery systems should be developed in order to reduce the burden of use in patients with fibrotic ILD.

10 questions met consensus for unsure and four questions did not meet consensus (supplementary table S1). Experts were unsure if pulmonary hypertension develops as a consequence of isolated exertional or nocturnal desaturation, or if oxygen therapy can slow the development of existing pulmonary hypertension. Experts were unsure if supplemental oxygen prevents adverse clinical outcomes such as hospitalisation or 
TABLE 1 Expert characteristics

\begin{tabular}{|c|c|c|}
\hline & Expert interview & Delphi survey \\
\hline Experts invited & 10 & 45 \\
\hline Response rate & $10 / 10(100)$ & 42/45 (93) \\
\hline Age years & $50.1 \pm 8.2$ & $50.5 \pm 8.5$ \\
\hline Female & $6(60)$ & $19(45)$ \\
\hline \multicolumn{3}{|l|}{ Country } \\
\hline Argentina & & $1(2.4)$ \\
\hline Australia & $1(10)$ & $5(11.9)$ \\
\hline Belgium & & $1(2.4)$ \\
\hline Brazil & & $1(2.4)$ \\
\hline Canada & & $4(9.5)$ \\
\hline Denmark & & $1(2.4)$ \\
\hline France & & $2(4.8)$ \\
\hline Germany & $1(10)$ & $2(4.8)$ \\
\hline Greece & & $1(2.4)$ \\
\hline Iceland & & $1(2.4)$ \\
\hline Italy & & $3(7.1)$ \\
\hline Japan & & $1(2.4)$ \\
\hline Mexico & & $1(2.4)$ \\
\hline The Netherlands & $1(10)$ & $2(4.8)$ \\
\hline Spain & $1(10)$ & $2(4.8)$ \\
\hline UK & $3(30)$ & $3(7.1)$ \\
\hline USA & $3(30)$ & $11(26.2)$ \\
\hline \multicolumn{3}{|l|}{ Occupation } \\
\hline Physician & $8(80)$ & $39(93)$ \\
\hline Nurse & 2 (20) & $3(7)$ \\
\hline Time in clinical practice years & $19.9 \pm 10$ & $19.1 \pm 9.3$ \\
\hline Time dedicated to ILD \% & $92 \pm 14$ & $77.3 \pm 22.3$ \\
\hline
\end{tabular}

acute exacerbation, or if oxygen therapy impacts survival in patients with fibrotic ILD. No consensus was reached on whether oxygen should be titrated according to symptom alleviation or whether it should be continued for physiological benefit in cases with no symptomatic improvement. In the sensitivity analysis, 15 out of $20(75 \%)$ items would still have met consensus for agreement or disagreement using a definition of $\geqslant 70 \%$ agreement. Five items would not have met consensus, including whether patients should undergo a screening test for nocturnal hypoxaemia, if oxygen should be titrated to achieve $\mathrm{SpO}_{2}>89 \%$ and regarding potential concerns about oxygen toxicity (table 2).

\section{Exertional desaturation threshold}

19 out of 42 (45\%) experts recommended oxygen below an $\mathrm{SpO}_{2}$ threshold of $<89 \%$, nine out of $42(21 \%)$ recommend it at a threshold of $<90 \%$ and nine out of $42(21 \%)$ at a threshold of $<85 \%$. A minority (four out of 42) did not recommend oxygen or did not use an $\mathrm{SpO}_{2}$ threshold and one recommended it below an $\mathrm{SpO}_{2}$ threshold of $<80 \%$ (figure 2). Throughout the survey, experts indicated that their recommended desaturation threshold would be considered in the context of symptoms, exercise tolerance and patient preference.

\section{Regional funding policies}

In the round 3 survey, nearly all experts (41 out of $42(98 \%)$ ) reported that supplemental oxygen would be funded in their region for fibrotic ILD patients with severe resting hypoxaemia (defined as $\mathrm{PaO}_{2}$ $<55 \mathrm{mmHg} / \mathrm{SPO}_{2}<89 \%$, or $\mathrm{PaO}_{2}<60 \mathrm{mmHg}$ and cor pulmonale and/or polycythaemia). For isolated exertional desaturation, access to oxygen funding varied depending on the $\mathrm{SpO}_{2}$ threshold and the presence or absence of symptoms attributable to the hypoxaemia (figure 3). The majority of experts reported that oxygen would be funded based on a desaturation threshold set at $<89 \%$, with broader access to funding based on lower desaturation thresholds and the presence of hypoxaemia-attributable symptoms. In contrast, most experts (30 out of $42(71 \%)$ ) reported no access to oxygen funding at an exertional desaturation threshold set at $<90 \%$, regardless of symptoms. Although not included in our clinical scenarios of exertional desaturation, some respondents noted that improved exercise tolerance must also be demonstrated prior to funding approval in their practice location. Notably, four out of 42 (9.5\%) and 10 


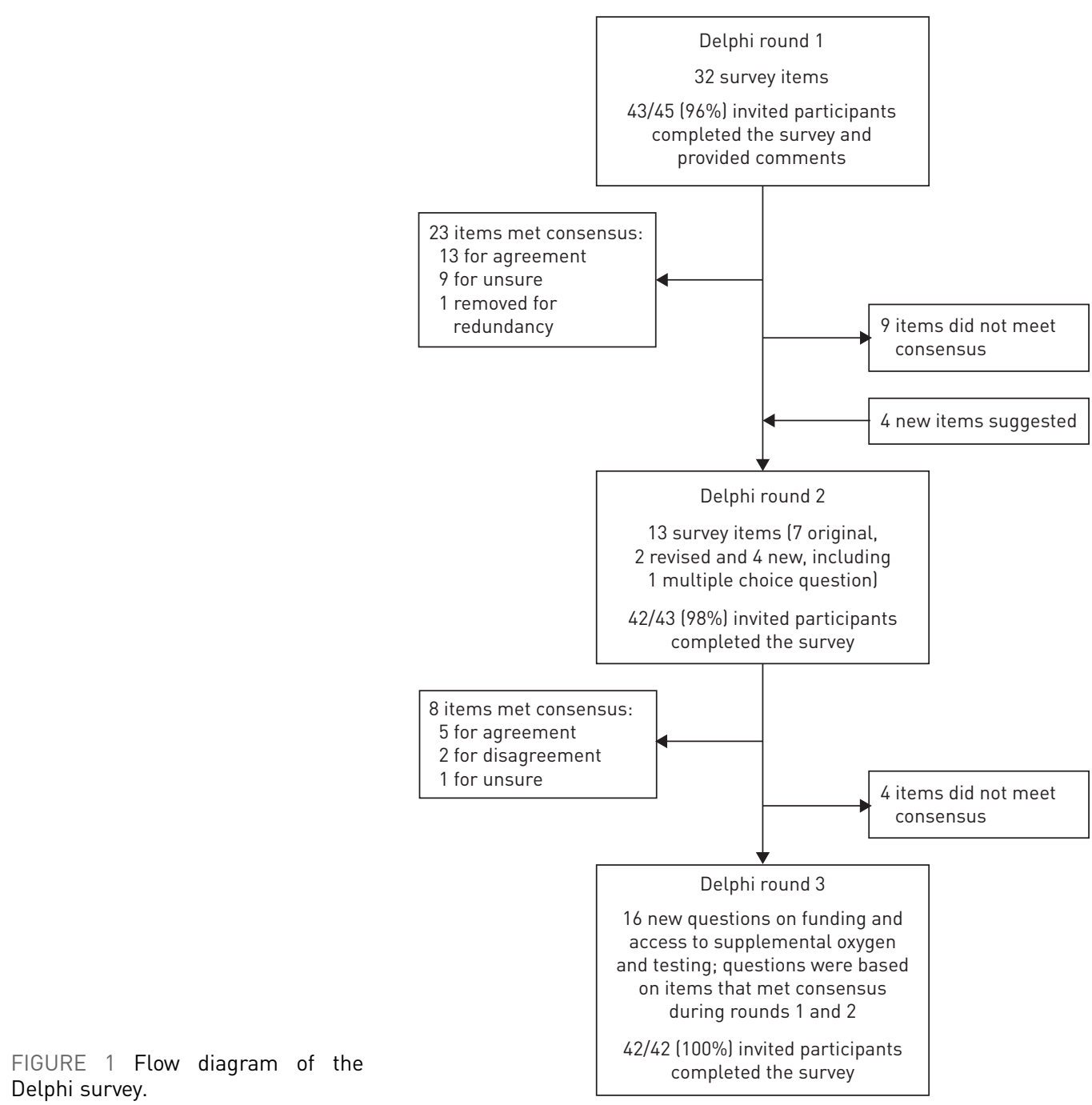

out of 42 (24\%) experts reported that no funding coverage would be available for an exertional $\mathrm{SpO}_{2}<80 \%$ with or without hypoxaemia-attributable symptoms, respectively. All experts reported routine access to physiological testing for exertional desaturation, e.g. 6-min walk test or shuttle walk test, at their ILD referral centre. The majority $(81 \%)$ reported routine access to overnight oximetry testing or sleep testing for assessment of nocturnal desaturation. Where $12 \%$ of experts practice, obese patients with fibrotic ILD are required to undergo a polysomnogram prior to being eligible for oxygen funding. As part of their regional oxygen funding algorithm, an arterial blood gas was reported as required by 19 out of 42 (45\%) in cases of resting desaturation and 11 out of $42(26 \%)$ in cases of isolated exertional desaturation. 40 out of 42 (95\%) experts reported that funding policies are consistent across different aetiologies of fibrotic ILD and only eight out of $42(19 \%)$ reported specific oxygen funding policies for fibrotic ILD patients with an anticipated survival of $<6$ months.

\section{Discussion}

In this study, our expert panel achieved consensus on 20 items that can be used to guide supplemental oxygen use in patients with fibrotic ILD. We further identified several areas where respondents were unsure or where consensus could not be met, suggesting future areas where research efforts should be focused. Finally, we identified discrepancies between scenarios where clinicians would recommend oxygen and poor patient access, based on regional funding criteria or testing requirements. These findings may be used to develop clinical practice recommendations and funding policies for supplemental oxygen for this patient population, pending more robust evidence. A proposed approach to the assessment and prescription of supplemental oxygen for patients with fibrotic ILD is presented in figure 4 .

Overall, experts agreed that supplemental oxygen is a safe therapeutic intervention that should be considered in conjunction with patient wishes and goals, and that newer portable systems should be 
TABLE 2 Items meeting consensus for agreement or disagreement

\section{Survey item}

Median (IQR) score from 1 to 5
Sensitivity analysis $\%$ agreement

\section{Indications for supplemental oxygen}

Supplemental oxygen should be recommended for fibrotic ILD patients with severe resting hypoxaemia $\left(\mathrm{PaO}_{2}<55 \mathrm{mmHg} / \mathrm{SpO}_{2}<89 \%\right.$, or $\mathrm{PaO}_{2}<60 \mathrm{mmHg}$ and cor pulmonale and/or polycythaemial

Supplemental oxygen should be recommended for fibrotic ILD patients with isolated exertional desaturation to $<80 \%$, regardless of symptoms

Recommendations for supplemental oxygen use should be consistent across different aetiologies of fibrotic ILD

Supplemental oxygen should be recommended for fibrotic ILD patients with nocturnal hypoxaemia in the absence of other causes such as sleep disordered breathing

Fibrotic ILD patients with isolated exertional hypoxaemia should be advised to use supplemental oxygen during sleep, without the need for nocturnal oximetry testing ${ }^{\#}$

\section{Goals of supplemental oxygen}

Supplemental oxygen should be titrated to achieve an oxygen saturation $>89 \%$ at all times

I recommend supplemental oxygen to improve physical symptoms (e.g. fatigue, decreased energy) in patients with fibrotic ILD and evidence of resting or exertional desaturation

I recommend supplemental oxygen to improve psychological symptoms le.g. cognition, mood) in patients with fibrotic ILD and evidence of resting or exertional desaturation

I recommend supplemental oxygen to improve dyspnoea in patients with fibrotic ILD and evidence of resting or exertional desaturation

I recommend supplemental oxygen to improve functional capacity in patients with fibrotic ILD and evidence of resting or exertional desaturation

I recommend supplemental oxygen to prevent deconditioning in patients with fibrotic ILD and evidence of resting or exertional desaturation

I recommend supplemental oxygen to improve physiological parameters such as oxygen delivery, cardiac output and arterial oxygen content in patients with fibrotic ILD and evidence of resting or exertional desaturation

\section{Other considerations for supplemental oxygen use}

Exertional hypoxaemia should be periodically evaluated by objective standardised testing including but not limited to 6MWT, CPET or shuttle walk in patients with fibrotic ILD

Prior to initiating and continuing supplemental oxygen prescription, the clinician should consider the balance of benefits versus burdens for individual patients with fibrotic ILD

Newer portable oxygen delivery systems should be developed in order to reduce the burden of use in patients with fibrotic ILD

Fibrotic ILD patients with isolated exertional hypoxaemia should undergo a screening test for nocturnal hypoxaemia

Oxygen toxicity is not a concern in most clinical scenarios where supplemental oxygen is prescribed for patients with fibrotic ILD

The development or worsening of exertional desaturation provides evidence of clinical deterioration in patients with fibrotic ILD

Resting hypoxaemia leads to the development of pulmonary hypertension in patients with fibrotic ILD

In patients prescribed supplemental oxygen for isolated exertional hypoxaemia, oxygen should be discontinued in those who do not report any improvement in symptoms or exercise capacity

ILD: interstitial lung disease; $\mathrm{PaO}_{2}$ : arterial oxygen tension; $\mathrm{SpO}_{2}$ : peripheral oxygen saturation; 6MWT: 6-min walk test; CPET: cardiopulmonary exercise test. ${ }^{\#}$ : consensus for disagreement.

developed to facilitate its use. There was consensus to recommend oxygen in cases of severe resting or exertional hypoxaemia and that nocturnal desaturation is an important clinical parameter. Experts agreed that the goals of oxygen use are multifaceted, primarily aiming to improve the patient experience, targeting symptoms and/or exercise tolerance. Experts recommend oxygen use in cases of symptomatic exertional desaturation, highlighting the different approach to oxygen prescription for patients with fibrotic ILD compared with patients with COPD. A large randomised trial found that supplemental oxygen did not improve survival, functional status or quality of life in stable COPD patients with moderate resting hypoxaemia or isolated exertional desaturation [25]. However, these findings should be extrapolated with caution to patients with fibrotic ILD, given the differing physiology and severity of exertional desaturation between these groups $[4,26]$. 


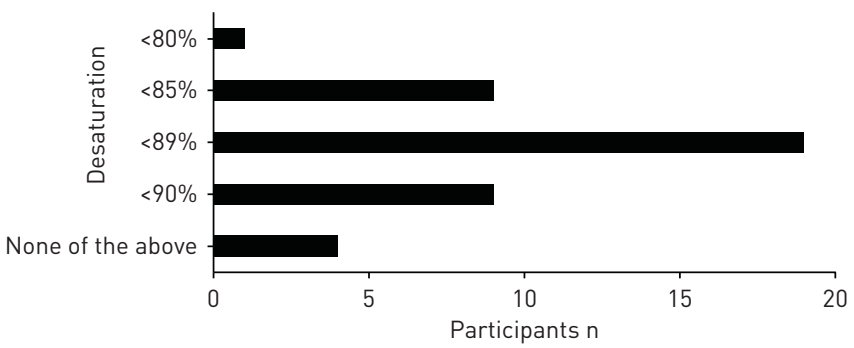

FIGURE 2 Expert-recommended exertional desaturation thresholds for supplemental oxygen use in patients with fibrotic interstitial lung disease. $n=42$ participants.

The only clinical scenario for which there was $100 \%$ strong agreement to recommend oxygen was in cases of severe resting hypoxaemia, consistent with published guidelines for ILD management [16, 17, 27]. Some country-specific recommendations suggest ambulatory oxygen for patients with ILD or IPF if breathlessness or exercise limitations exist in the context of exertional desaturation [17, 27]. In our study, most experts recommended oxygen below an exertional desaturation threshold between $85 \%$ and $89 \%$, and particularly when patients have symptoms or exercise intolerance that improved with oxygen therapy. Given the dynamic and effort-dependent nature of exertional desaturation, expert commentary suggested that a single measured $\mathrm{SpO}_{2}$ in isolation provides inadequate data to guide oxygen prescription. Desaturation level should likely be considered in conjunction with symptoms, exercise tolerance and possibly with evidence of improvement in these parameters with oxygen therapy.

All clinical decisions, including the use of supplemental oxygen, should be made in partnership with patients and, where appropriate, caregivers. Prior qualitative and mixed-methods studies have addressed the goals of oxygen use from both the healthcare provider and patient perspective [28-30]. The summarised goals appear consistent, primarily focused on improving symptoms and exercise tolerance. The current study focused uniquely on healthcare providers' opinions regarding the indications and goals of oxygen for patients with fibrotic ILD. We wanted to understand the recommendations being made by clinicians, as this is inexorably related to how information is presented to patients. The discussion of anticipated benefits and potential risks or complications of therapy can influence a patient's decision to accept or decline treatment in shared decision-making models [31]. In the absence of definitive evidence, we hope that our findings can provide a framework for such discussions. Further studies addressing internationally diverse patient and caregiver perspectives would add important data guiding the role of

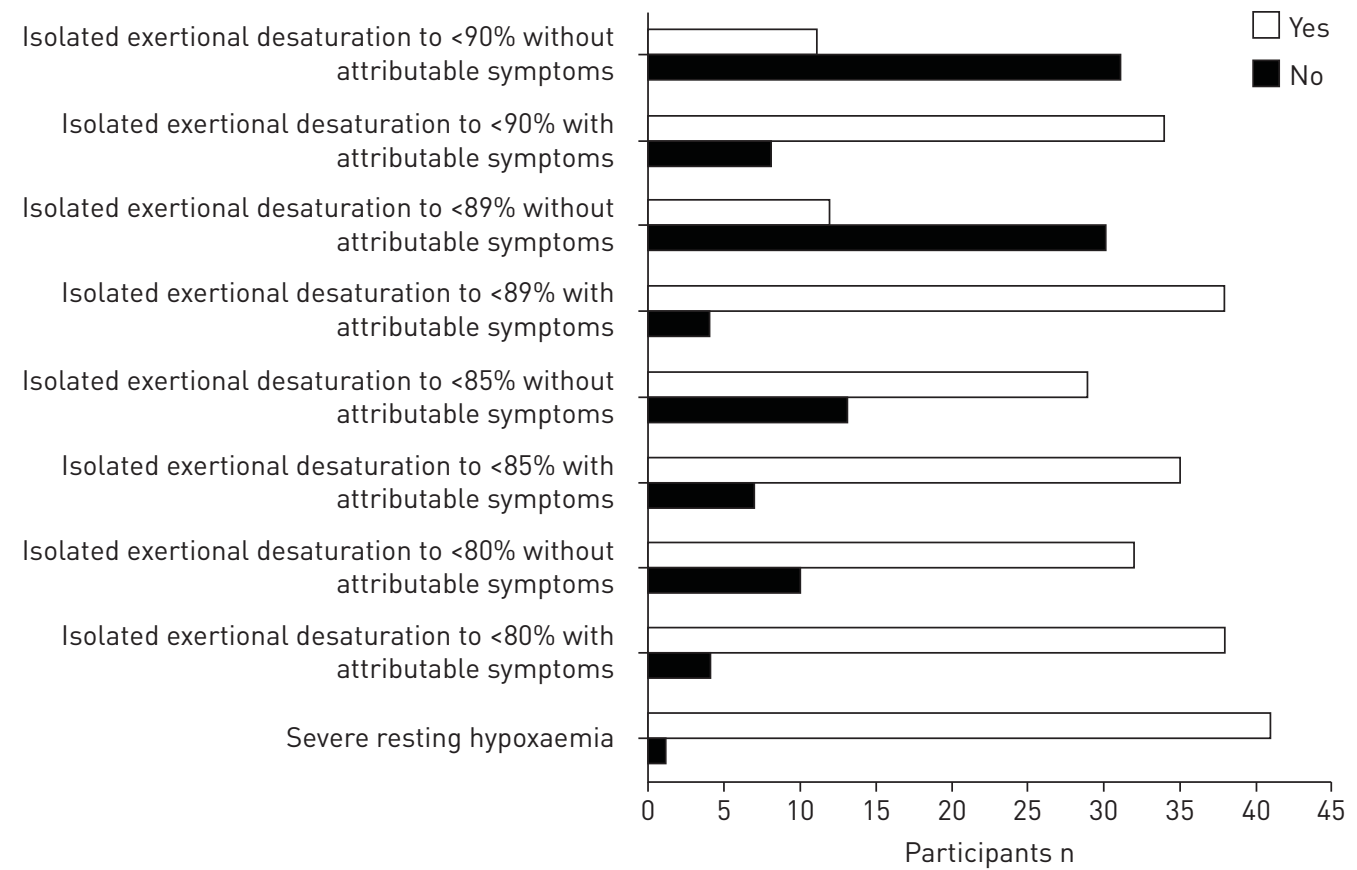

FIGURE 3 Regional oxygen funding coverage for patients with fibrotic interstitial lung disease, based on specific exertional desaturation criteria. 


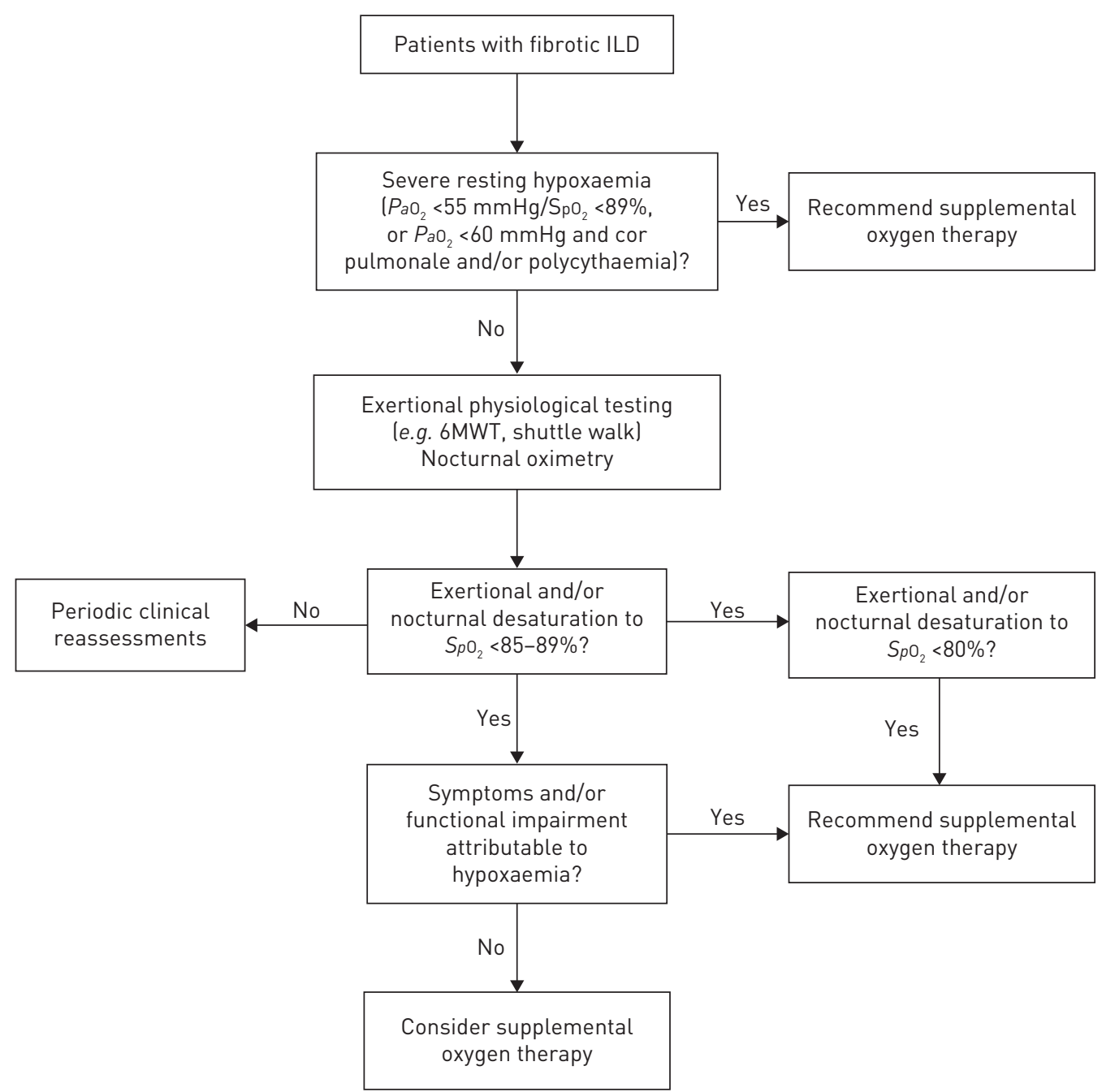

FIGURE 4 Proposed algorithm for assessment and prescription of oxygen. Decisions regarding supplemental oxygen use should be made with consideration of the benefits and risks, as well as patient values and preferences. ILD: interstitial lung disease; $\mathrm{PaO}_{2}$ : arterial oxygen tension; $\mathrm{SpO}_{2}$ : peripheral oxygen saturation; 6MWT: 6-min walk test.

oxygen. Despite differences in clinical practice patterns among our experts, there was a consensus that clinicians should consider the balance of benefits versus burdens of supplemental oxygen for individual patients with fibrotic ILD, an essential point underlying all work on this topic.

The Delphi technique is a validated methodology used in healthcare research to develop consensus-based recommendations when robust evidence is lacking. Through iterative questionnaires, a group of experts provides their opinion after considering the collective responses of prior rounds, while maintaining anonymity $[23,24]$. We believe that the Delphi technique was a justified research tool to identify current best practices in supplemental oxygen use among patients with fibrotic ILD. Based on expert input representing 17 countries, access to oxygen funding appears heterogeneous by practice location. In some regions, there are few barriers to oxygen funding, while in others oxygen is only accessible if patients pay for the equipment themselves or if they qualify via predefined testing algorithms. One-quarter of experts reported that no oxygen funding is available for patients with exertional desaturation $<80 \%$ in the absence of attributable symptoms, highlighting the heterogeneity of access to this therapy in different practice areas. Standardising the clinical indications for oxygen may make access more equitable across regions, but this is a complex goal given the variability in healthcare systems and oxygen reimbursement policies worldwide. Furthermore, technological advances should be prioritised to reduce the burden of portable oxygen devices for advanced lung disease patients [32, 33].

This study has limitations. First, our participant selection strategy was not based on predefined or publication-based criteria. Rather, we sought to achieve representative diversity of experience and opinion 
from a large pool of potential international ILD experts. We believe this is particularly important with our study question, given the lack of high-quality evidence. In support of our expert selection approach are the very high participation and survey completion rates. Participants from the Asian and African continents are under-represented in this survey, and future work should aim to better understand clinical practice patterns in these regions. Based on the survey design of this study, we were unable to further qualify the expert responses to define specific clinical scenarios in which supplemental oxygen would be used. Given the unique circumstances of each patient encounter, this was not feasible. Finally, the consensus among experts does not necessarily equate to scientific truth or clinical validity and lack of consensus highlights differences in perspectives regarding complex issues. A Delphi survey is not a substitute for rigorous prospective studies and many of the questions posed herein, including those meeting consensus, should be further addressed in randomised trials of oxygen therapy, where possible.

\section{Conclusions}

An international group of ILD experts achieved consensus on 20 items to guide supplemental oxygen use in patients with fibrotic ILD. Desaturation severity, hypoxaemia-attributable symptoms, exercise tolerance and patient preference should be considered when recommending oxygen. While there is a lack of evidence to guide clinical recommendations, oxygen is an important component of patient management and well-designed prospective studies are urgently needed to answer outstanding questions. The current data may provide a framework to guide clinical decision making and funding policy for oxygen therapy in this patient population, pending the availability of high-quality evidence.

Acknowledgements: We would like to sincerely thank the $\mathrm{O}_{2}$ Delphi Collaborators who completed this survey for their time and invaluable contributions to this work: Katerina M. Antoniou, Deborah Assayag, Elisabeth Bendstrup, Francesco Bonella, Kevin K. Brown, Bruno Crestani, Tamera J. Corte, Vincent Cottin, Sonye K. Danoff, Jolene H. Fisher, Kevin R. Flaherty, Ian Glaspole, Nicole S. Goh, Jan C. Grutters, Gunnar Gudmundsson, Susan S. Jacobs, Leticia Kawano-Dourado, Yet H. Khor, Martin Kolb, Yasuhiro Kondoh, Michael Kreuter, Lisa H. Lancaster, Kathleen O. Lindell, Toby M. Maher, Mariano Mazzei, Maria Molina-Molina, David J. Lederer, Christine F. McDonald, Carlos A.C. Pereira, Elizabeth A. Renzoni, Anne-Marie Russell, Christopher J. Ryerson, Jay H. Ryu, Moises Selman, Paolo Spagnolo, Mary E. Strek, Jeffrey J. Swigris, Sara Tomassetti, Claudia Valenzuela, Carlo Vancheri, Athol U. Wells, Marlies S. Wijsenbeek, Paul J. Wolters and Wim Wuyts.

Author contributions: R.K. Lim and K.A. Johannson conceived of the study; all authors made substantial contributions to the study design, data analysis and data interpretation; R.K. Lim and K.A. Johannson drafted the manuscript; all authors critically appraised and approved the final version of the submitted manuscript.

Conflict of interest: R.K. Lim has nothing to disclose. C. Humphreys has nothing to disclose. J. Morisset has participated on advisory boards and provided consultation for Hoffman-La Roche Ltd and Boehringer Ingelheim, outside the submitted work. A.E. Holland reports nonfinancial support (equipment for research) from Air Liquide, outside the submitted work. K.A. Johannson reports grants from University of Calgary Division of Respiratory Medicine Fellowship Research Fund, during the conduct of the study; personal fees from Hoffman-La Roche Ltd, Boehringer Ingelheim and Theravance, grants from UCB Biopharma SPRL, Chest Foundation and University of Calgary Cumming School of Medicine, outside the submitted work.

Support statement: This study was supported by funding from the Division of Respirology, University of Calgary, Calgary, AB, Canada. Funding information for this article has been deposited with the Crossref Funder Registry.

\section{References}

1 Risk C, Epler GR, Gaensler EA. Exercise alveolar-arterial oxygen pressure difference in interstitial lung disease. Chest 1984; 85: 69-74.

2 Agusti AG, Roca J, Rodriguez-Roisin R, et al. Different patterns of gas exchange response to exercise in asbestosis and idiopathic pulmonary fibrosis. Eur Respir J 1988; 1: 510-516.

3 Agusti AG, Roca J, Gea J, et al. Mechanisms of gas-exchange impairment in idiopathic pulmonary fibrosis. Am Rev Respir Dis 1991; 143: 219-225.

4 Du Plessis JP, Fernandes S, Jamal R, et al. Exertional hypoxemia is more severe in fibrotic interstitial lung disease than in COPD. Respirology 2018; 23: 392-398.

5 Lama VN, Flaherty KR, Toews GB, et al. Prognostic value of desaturation during a 6-minute walk test in idiopathic interstitial pneumonia. Am J Respir Crit Care Med 2003; 168: 1084-1090.

6 Timmer SJ, Karamzadeh AM, Yung GL, et al. Predicting survival of lung transplantation candidates with idiopathic interstitial pneumonia: does $\mathrm{PaO}_{2}$ predict survival? Chest 2002; 122: 779-784.

7 Swigris JJ, Brown KK, Abdulqawi R, et al. Patients' perceptions and patient-reported outcomes in progressive-fibrosing interstitial lung diseases. Eur Respir Rev 2018; 27: 180075.

8 Bye PT, Anderson SD, Woolcock AJ, et al. Bicycle endurance performance of patients with interstitial lung disease breathing air and oxygen. Am Rev Respir Dis 1982; 126: 1005-1012.

9 Frank RC, Hicks S, Duck AM, et al. Ambulatory oxygen in idiopathic pulmonary fibrosis: of what benefit? Eur Respir J 2012; 40: 269-270.

10 Visca D, Montgomery A, de Lauretis A, et al. Ambulatory oxygen in interstitial lung disease. Eur Respir J 2011; 38: 987-990.

11 Bell EC, Cox NS, Goh N, et al. Oxygen therapy for interstitial lung disease: a systematic review. Eur Respir Rev 2017; 26: 160080. 
12 Douglas WW, Ryu JH, Schroeder DR. Idiopathic pulmonary fibrosis: impact of oxygen and colchicine, prednisone, or no therapy on survival. Am J Respir Crit Care Med 2000; 161: 1172-1178.

13 Visca D, Mori L, Tsipouri V, et al. Effect of ambulatory oxygen on quality of life for patients with fibrotic lung disease (AmbOx): a prospective, open-label, mixed-method, crossover randomised controlled trial. Lancet Respir Med 2018; 6: 759-770.

14 Nocturnal Oxygen Therapy Trial Group. Continuous or nocturnal oxygen therapy in hypoxemic chronic obstructive lung disease: a clinical trial. Ann Intern Med 1980; 93: 391-398.

15 Medical Research Council Working Party. Long term domiciliary oxygen therapy in chronic hypoxic cor pulmonale complicating chronic bronchitis and emphysema. Report of the Medical Research Council Working Party. Lancet 1981; 1: 681-686.

16 Raghu G, Collard HR, Egan JJ, et al. An official ATS/ERS/JRS/ALAT statement: idiopathic pulmonary fibrosis: evidence-based guidelines for diagnosis and management. Am J Respir Crit Care Med 2011; 183: 788-824.

17 Bradley B, Branley HM, Egan JJ, et al. Interstitial lung disease guideline: the British Thoracic Society in collaboration with the Thoracic Society of Australia and New Zealand and the Irish Thoracic Society. Thorax 2008; 63: Suppl. 5, v1-v58.

18 Morisset J, Ryerson CJ, Johannson KA. Oxygen prescription in interstitial lung disease: 2.5 billion years in the making. Ann Am Thorac Soc 2017; 14: 1755-1756.

19 Johannson KA, Pendharkar SR, Mathison K, et al. Supplemental oxygen in interstitial lung disease: an art in need of science. Ann Am Thorac Soc 2017; 14: 1373-1377.

20 Humphreys CJ, Lim R, Morisset J, et al. Supplemental oxygen use in patients with fibrotic interstitial lung disease: an international qualitative study. Am J Respir Crit Care Med 2019; 199: A4771.

21 Graneheim UH, Lundman B. Qualitative content analysis in nursing research: concepts, procedures and measures to achieve trustworthiness. Nurse Educ Today 2004; 24: 105-112.

22 Cavanagh S. Content analysis: concepts, methods and applications. Nurse Res 1997; 4: 5-16.

23 Junger S, Payne SA, Brine J, et al. Guidance on Conducting and REporting DElphi Studies (CREDES) in palliative care: recommendations based on a methodological systematic review. Palliat Med 2017; 31: 684-706.

24 Diamond IR, Grant RC, Feldman BM, et al. Defining consensus: a systematic review recommends methodologic criteria for reporting of Delphi studies. J Clin Epidemiol 2014; 67: 401-409.

25 Albert RK, Au DH, Blackford AL, et al. A randomized trial of long-term oxygen for COPD with moderate desaturation. N Engl J Med 2016; 375: 1617-1627.

26 Swigris J. Caution against extrapolating results from the trial of long-term oxygen for chronic obstructive pulmonary disease. Ann Am Thorac Soc 2017; 14: 296.

27 Funke-Chambour M, Azzola A, Adler D, et al. Idiopathic pulmonary fibrosis in Switzerland: diagnosis and treatment. Respiration 2017; 93: 363-378.

28 Khor YH, Goh NS, McDonald CF, et al. Oxygen therapy for interstitial lung disease: a mismatch between patient expectations and experiences. Ann Am Thorac Soc 2017; 14: 888-895.

29 Khor YH, Goh NSL, McDonald CF, et al. Oxygen therapy for interstitial lung disease: physicians' perceptions and experiences. Ann Am Thorac Soc 2017; 14: 1772-1778.

30 Graney BA, Wamboldt FS, Baird S, et al. Looking ahead and behind at supplemental oxygen: a qualitative study of patients with pulmonary fibrosis. Heart Lung 2017; 46: 387-393.

31 Epstein RM, Alper BS, Quill TE. Communicating evidence for participatory decision making. JAMA 2004; 291: 2359-2366.

32 Jacobs SS, Lederer DJ, Garvey CM, et al. Optimizing Home Oxygen Therapy. An Official American Thoracic Society Workshop Report. Ann Am Thorac Soc 2018; 15: 1369-1381.

33 Ramadurai D, Riordan M, Graney B, et al. The impact of carrying supplemental oxygen on exercise capacity and dyspnoea in patients with interstitial lung disease. Respir Med 2018; 138: 32-37. 